\title{
Clinical Study \\ Previous Leisure-Time Physical Activity Dose Dependently Decreases Ischemic Stroke Severity
}

\author{
Dominique Deplanque, ${ }^{1,2}$ Isabelle Masse, ${ }^{1,2}$ Christian Libersa, ${ }^{1,2}$ \\ Didier Leys, ${ }^{3}$ and Régis Bordet ${ }^{1}$ \\ ${ }^{1}$ Department of Medical Pharmacology, Faculty of Medicine, University Lille-North of France, 1 Place Verdun, 59045 Lille, France \\ ${ }^{2}$ Clinical Investigation Centre, University Lille-North of France, CIC 9301 INSERM-CHRU, 59037 Lille, France \\ ${ }^{3}$ Neurology Department and Stroke Unit, University Lille-North of France, Lille University Hospital, 59037 Lille, France
}

Correspondence should be addressed to Régis Bordet, bordet@univ-lille2.fr

Received 11 May 2011; Revised 30 June 2011; Accepted 7 July 2011

Academic Editor: Julie A. Bernhardt

Copyright (C) 2012 Dominique Deplanque et al. This is an open access article distributed under the Creative Commons Attribution License, which permits unrestricted use, distribution, and reproduction in any medium, provided the original work is properly cited.

In the present subanalysis of a cross-sectional study showing the favorable effect of prior transient ischemia, leisure-time physical activity, and lipid-lowering drug therapy on stroke severity, we aimed to evaluate whether previous physical activity was dose dependently associated to minor stroke (NIHSS 0-3) and to identify possible underlying factors. Among 362 consecutive patients, less severe stroke was related to weekly exercise duration prior to stroke (no exercise: $36.1 \%$; <2 hours: $49.3 \%$; $2-5$ hours: $58.8 \%$; $>5$ hours: $64.0 \% ; P=0.003$ ). Only weak and moderate exercise practices were protective (weak: $50.0 \%$; moderate: $79.3 \%$; heavy: $22.2 \% ; P<0.0001)$. Such a beneficial effect was observed independently of age and was associated with a trend to a lower frequency of arterial hypertension, alcohol abuse, and a better metabolic profile. Besides other therapeutic approaches, physical activity may be a simple way to decrease cerebral ischemia severity.

\section{Introduction}

Meta-analyses of epidemiologic studies have consistently suggested the risk reduction of both ischemic and hemorrhagic strokes when practicing regular physical activity [13]. Such a preventive effect, that may be more important in men than in women [3], could be very impressive, since the relative risk of stroke incidence and mortality could be reduced from $25 \%$ (cohort studies) to up to $64 \%$ (case-control studies) in active individuals as compared to nonactive counterparts $[1,2]$. Several plausible mechanisms by which physical activity might reduce stroke risk has been discussed as reducing blood pressure or improving lipid profiles, endothelial function, and haemostatic and inflammatory parameters [1]. Beyond the decrease of stroke incidence, it could also be postulated that physical activity could lead to the reduction of stroke severity, since both experimental and clinical data provide some arguments about a possible preventive neuroprotection [4-8].
In a previously published study [6], we showed that previous leisure-time physical activity as well as prior transient ischemic attack and lipid-lowering drugs therapy are independent predictors of lesser stroke severity and better short-term outcome. In this context, at least 2 other studies provide similar results, but little is known about the possible underlying mechanisms $[7,8]$. In the present analysis, we aimed to focus on the impact of previous leisuretime physical activity on initial stroke severity. To provide some explanations about such a possible beneficial effect, we analyzed patients' clinical and biological profiles. Taking into account both the duration and the type of previous physical activity and adjusting on a number of possible confounders, we evaluated whether previous physical activity was dose dependently associated to minor stroke.

\section{Materials and Methods}

2.1. Study Population. Population characteristics and methodological design of the study have been already published 
and extensively detailed [6]. Briefly, we studied consecutive patients with an acute ischemic stroke who were admitted to the stroke unit of the Lille University Hospital, France. The inclusion criteria were (1) clinical symptoms and signs attributable to cerebral ischemia of $<24$-hour duration (onset of symptoms was defined as the last time the patient was free of any symptoms) and (2) acute or subacute cerebral computed tomography or magnetic resonance with changes typical of acute hemispheric cerebral infarction.

2.2. Main Data Recorded. For each patients, we recorded the following data: demographics, vascular risk factors, previous treatments, severity of the neurological deficit by the $\mathrm{Na}$ tional Institutes of Health Stroke Scale (NIHSS), blood pressure, body temperature, fasting glycemia, cholesterol, triglycerides, and fibrinogen at admission, the presence, weekly duration (less than 2 hours, between 2 and 5 hours, more than 5 hours), and intensity (weak, moderate, or heavy) of previous leisure-time physical activity $[6,9]$, and cognitive status with the mini mental state when assessable and functional outcome as measured with the modified Rankin scale (mRS) and the Barthel index (BI) at 8 days. An NIHSS of 0 to 3 at admission was considered as minor stroke, while an mRS of 0 to 1 or BI of 95 to 100 at 8 days was considered favorable short-term outcome.

2.3. Statistical Analysis. To precisely analyze the impact of previous regular physical activity on the occurrence of a minor stroke and to determine the possible underlying factors, we performed univariate analysis comparing patients with previous physical activity and patients without using the $\chi 2$ test with Yates' correction or Fisher's exact test when appropriate, odds ratio (OR) with $95 \%$ confidence intervals (CI), and the unpaired $t$-test or Mann-Whitney $U$ test. In a second step, we performed stepwise logistic regression analyses with NIHSS 0 to 3 as dependent variable and previous leisure-time physical activity, physical activity duration, or physical activity intensity as main criteria. Other covariates included in the model were those assessable at admission. These variables were selected from the results of previous univariate analyses with a $P<0.25$ level as screening criterion [6]. Colinearity between variables (defined as $r>$ 0.6) was also excluded. Adjusted OR and their 95\% CI were then calculated. Logistic regression analyses were also performed to measure the possible interactions between previous leisure-time physical activity and variables selected to adjust the model. Finally, we also compared patients with previous physical activity and patients without by taking into account different age subgroups ( $<65$ years, $65-75$ years, $>75$ years). All the statistical procedures were computed using the SPSS-15.0 for windows package.

\section{Results}

Main characteristics of the study population have been extensively described before [6]. Of the 362 patients (median age 70, range 16-97) included in the study, 167 (46.1\%) were men, and $132(36.5 \%)$ had a regular physical activity before stroke. According to predefined criteria $[6,9]$, the intensity of previous leisure-time physical activity was considered as weak in 94 patients $(26.0 \%)$, moderate in $29(8.0 \%)$, and heavy in $9(2.5 \%)$ when weekly duration was less than 2 hours in $73(20.2 \%)$, between 2 and 5 hours in $34(9.4 \%)$ and over 5 hours in $25(6.9 \%)$. Patients with previous leisure-time physical activity were more likely to have a minor stroke or a better short-term outcome, but the proportion of TIA and brain infarction remained similar (Table 1). Concerning possible underlying factors, patients with a previous physical activity were more likely to be younger and men (Table 1). Apart from smoking, they also were less likely to have vascular risk factors and to receive chronic treatments (Table 1). Physiological and biological parameters at admission were also more likely to have better values, while stroke etiologies were more likely to be cervical artery dissections rather than the consequence of cardiac embolism or atherosclerosis disease (Table 1).

Adjusting on age, sex, arterial hypertension, diabetes, smoking, previous ischemic or congestive heart disease, and peripheral arteriopathy (according to colinearity prior treatments were not included in the model), we found a dose-dependent effect of physical activity duration on the initial stroke severity (Figure 1). The proportion of patients admitted for a minor stroke increased in parallel to the weekly duration of previous physical activity (no exercise: 83 (36.1\%); <2 hours: 36 (49.3\%); 2-5 hours: 20 (58.8\%); $>5$ hours: $16(64.0 \%) ; P=0.003)$. In terms of intensity, the favorable effect of previous physical activity was observed in patients who did weak or moderate exercises when heavy exercise may lead to deleterious consequences (proportion of minor stroke; weak: 47 (50.0\%); moderate: 23 (79.3\%); heavy: $2(22.2 \%) ; P<0.0001)$ (Figure 1). Furthermore, none of the interactions between previous leisure-time physical activity and covariates used to adjust the model was positive (result not shown).

Finally, the favorable effect of previous physical activity on initial stroke severity or short-term outcome remained whatever the age subgroups (Table 2). In this way, there also was a trend for an association between previous physical activity and a lower frequency of deleterious conditions such as arterial hypertension and alcohol abuse, while the metabolic profile of such patients was better (Table 2).

\section{Discussion}

The present study confirms that previous regular physical activity could lead to a possibly dose-dependent decrease in stroke severity. While both weak and moderate regular physical activities contribute to this possible neuroprotective effect, practicing heavy exercise may be deleterious. Analyzing all patients' characteristics, the protective effect of previous regular physical activity remains whatever the age group and may be related in part to a better vascular or metabolic profile.

The present analysis confirms the benefit of previous regular physical activity on stroke initial severity and outcome [6-8]. One of the most important points underlined 
TABLE 1: Main characteristics according to physical activity status before stroke.

\begin{tabular}{|c|c|c|c|c|c|}
\hline \multicolumn{6}{|c|}{ Previous physical activity } \\
\hline & Yes $(n=132)$ & No $(n=230)$ & OR & $95 \% \mathrm{CI}$ & $P$ \\
\hline \multicolumn{6}{|l|}{ Demographic characteristics } \\
\hline Age* & $56(16-86)$ & $73(25-97)$ & \multirow{2}{*}{1.62} & \multirow{2}{*}{$1.05-2.50$} & $<0.0001$ \\
\hline Men & $71(53.8 \%)$ & $96(41.7 \%)$ & & & 0.027 \\
\hline \multicolumn{6}{|l|}{ Medical history } \\
\hline Arterial hypertension & $56(42.4 \%)$ & $161(70.0 \%)$ & 0.32 & $0.20-0.49$ & $<0.0001$ \\
\hline Diabetes & $23(17.4 \%)$ & $65(28.3 \%)$ & 0.54 & $0.31-0.91$ & 0.022 \\
\hline Smoking & $47(35.6 \%)$ & $55(23.9 \%)$ & 1.76 & $1.10-2.81$ & 0.017 \\
\hline Ischemic heart disease & $15(11.4 \%)$ & $46(20.0 \%)$ & 0.51 & $0.27-0.96$ & 0.041 \\
\hline Congestive heart disease & $6(4.5 \%)$ & $40(17.4 \%)$ & 0.23 & $0.09-0.55$ & $<0.0001$ \\
\hline Peripheral arteriopathy & $4(3.0 \%)$ & $20(8.7 \%)$ & 0.33 & $0.11-0.99$ & 0.047 \\
\hline \multicolumn{6}{|l|}{ Previous treatment } \\
\hline Antiplatelet therapy & $35(26.5 \%)$ & $88(38.3 \%)$ & 0.58 & $0.36-0.93$ & 0.028 \\
\hline ACE inhibitors & $21(15.9 \%)$ & $63(27.4 \%)$ & 0.50 & $0.29-0.87$ & 0.014 \\
\hline Angiotensin receptor antagonists & $9(6.8 \%)$ & $35(15.2 \%)$ & 0.41 & $0.19-0.88$ & 0.019 \\
\hline Diuretic & $26(19.7 \%)$ & $80(34.8 \%)$ & 0.46 & $0.28-0.77$ & 0.003 \\
\hline Nitrate derivatives & $4(3.0 \%)$ & $27(11.7 \%)$ & 0.23 & $0.08-0.69$ & 0.003 \\
\hline Digoxin & $3(2.3 \%)$ & $24(10.4 \%)$ & 0.20 & $0.06-0.68$ & 0.003 \\
\hline Other antiarrhythmic drugs & $4(3.0 \%)$ & $25(10.9 \%)$ & 0.26 & $0.09-0.76$ & 0.008 \\
\hline Index stroke & & & & & 0.273 \\
\hline Cerebral infarction & $102(77.3 \%)$ & $189(82.8 \%)$ & 0.74 & $0.43-1.25$ & \\
\hline TIA & $30(29.4 \%)$ & $41(17.8 \%)$ & 1.36 & $0.80-2.31$ & \\
\hline Stroke etiology & & & & & 0.001 \\
\hline Atherosclerosis disease & $19(14.4 \%)$ & $54(23.5 \%)$ & 0.55 & $0.31-0.97$ & \\
\hline Cardioembolic stroke & $21(15.9 \%)$ & $65(28.3 \%)$ & 0.48 & $0.28-0.83$ & \\
\hline Lacunar stroke & $22(16.7 \%)$ & $30(13.0 \%)$ & 1.33 & $0.73-2.43$ & \\
\hline Cervical artery dissection & $15(11.4 \%)$ & $7(3.0 \%)$ & 4.08 & $1.61-10.33$ & \\
\hline Undetermined etiology & $54(40.9 \%)$ & $74(32.2 \%)$ & 1.46 & $0.93-2.28$ & \\
\hline \multicolumn{6}{|l|}{ Stroke severity and outcome } \\
\hline NIHSS at admission* & $3(0-27)$ & $5(0-35)$ & & & 0.002 \\
\hline Minor stroke (NIHSS 0-3 at admission) & $72(54.5 \%)$ & $83(36.1 \%)$ & 2.13 & $1.37-3.29$ & 0.001 \\
\hline Barthel $95-100$ at 8 days & $100(75.8 \%)$ & $72(31.3 \%)$ & 3.59 & $2.23-5.79$ & $<0.0001$ \\
\hline Rankin $0-1$ at 8 days & $76(57.6 \%)$ & $96(41.7 \%)$ & 1.89 & $1.23-2.93$ & 0.003 \\
\hline Mini mental state ${ }^{\dagger}$ & $27(5)$ & $24(10)$ & & & 0.003 \\
\hline \multicolumn{6}{|l|}{ Physiological parameters $^{\ddagger}$} \\
\hline Systolic Blood Pressure (mm Hg) & $142(27)$ & $151(30)$ & & & 0.003 \\
\hline Fibrinogen $(\mu \mathrm{mol} / \mathrm{L})$ & $10.41(2.70)$ & $11.70(3.27)$ & & & $<0.0001$ \\
\hline Glycemia (mmol/L) & $5.94(1.83)$ & $6.55(2.55)$ & & & 0.017 \\
\hline Total cholesterol (mmol/L) & $5.03(1.01)$ & $5.39(1.26)$ & & & 0.005 \\
\hline LDL cholesterol (mmol/L) & $2.89(0.85)$ & $3.17(1.03)$ & & & 0.005 \\
\hline
\end{tabular}

OR: odds ratio; CI: confidence interval; ACE: angiotensin converting enzyme. ${ }^{*}$ Result is reported as median (range). ${ }^{\dagger}$ Result is reported as mean (SD) in patients for whom the test was assessable. ${ }^{\ddagger}$ All results are given as mean (SD). All other variables did not differ between both groups.

here is the possible dose-dependent effect according to the weekly duration of physical exercise [6,7]. Indeed, our study suggests an exercise duration-dependent neuroprotective effect of physical activity. Besides the duration, the type and level of physical exercise seem also particularly important. Patients with regular heavy exercises may be exposed to more severe stroke, but the small number of subjects in this subgroup did not allow a definitive conclusion. A recently published study also underlined that regular physical exercise may be protective, while only practicing bout of physical exercise may contribute to the occurrence of an ischemic stroke [10]. The neuroprotective effect of previous regular physical activity may also contribute to the apparent decrease in stroke incidence, by reducing cerebral infarct size to levels that are not symptomatic [1-3].

Our findings support the results of experimental studies in which physical activity before ischemia induced neuroprotection $[4,5]$. In rats, both voluntary running and 


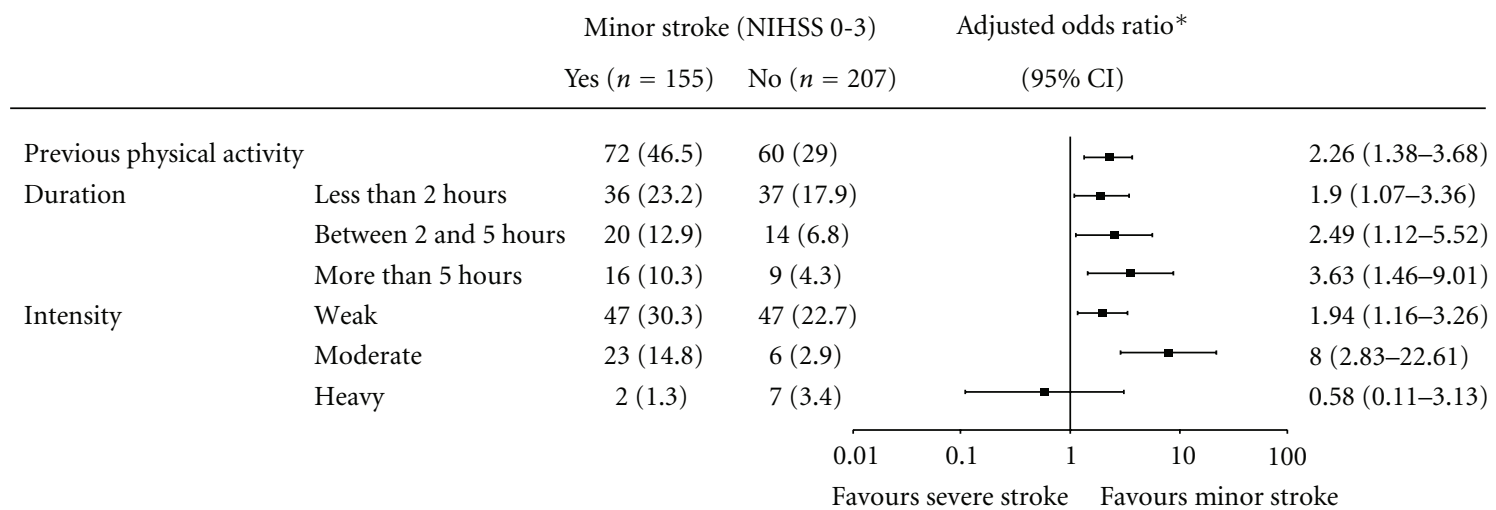

${ }^{*}$ Results were adjusted according to age, sex, and the presence of arterial hypertension, diabetes, smoking, previous ischemic or congestive heart disease, and peripheral arteriopathy (according to colinearity prior treatments were not included in the model).

FIGURE 1: Impact of previous leisure-time physical activity on initial stroke severity.

TABLe 2: Main characteristics associated to previous leisure-time physical activity according to age.

\begin{tabular}{|c|c|c|c|c|c|}
\hline \multicolumn{6}{|c|}{ Previous physical activity } \\
\hline & Yes $(n=132)$ & No $(n=230)$ & OR & CI 95\% & $P$ \\
\hline Age $<60$ years & $n=71$ & $n=51$ & & & \\
\hline Alcohol abuse & $7(9.9 \%)$ & $15(29.4 \%)$ & 0.26 & $0.10-0.71$ & 0.006 \\
\hline Arterial hypertension & $18(25.4 \%)$ & $21(41.2 \%)$ & 0.49 & $0.22-1.05$ & 0.065 \\
\hline Peripheral arteriopathy & - & $3(5.9 \%)$ & NA & & 0.071 \\
\hline Minor stroke (NIHSS 0-3 at admission) & $39(54.9 \%)$ & $16(31.4)$ & 2.67 & $1.25-5.68$ & 0.011 \\
\hline Barthel $95-100$ at 8 days & $62(87.3 \%)$ & $32(62.7 \%)$ & 4.09 & $1.66-10.10$ & 0.003 \\
\hline Age 60-75 years & $n=40$ & $n=85$ & & & \\
\hline Men & $26(65.0 \%)$ & $34(40.0 \%)$ & 2.79 & $1.27-6.10$ & 0.012 \\
\hline Minor stroke (NIHSS $0-3$ at admission) & $22(55.0 \%)$ & $30(35.3 \%)$ & 2.24 & $1.04-4.83$ & 0.037 \\
\hline Glycemia $(\mathrm{mmol} / \mathrm{L})^{*}$ & $6.16(1.44)$ & $7.05(2.83)$ & & & 0.061 \\
\hline LDL cholesterol $(\mathrm{mmol} / \mathrm{L})^{*}$ & $2.92(0.72)$ & $3.30(0.93)$ & & & 0.017 \\
\hline Age $>75$ years & $n=21$ & $n=94$ & & & \\
\hline Arterial hypertension & $11(52.4 \%)$ & $77(81.9 \%)$ & 0.24 & $0.09-0.67$ & 0.004 \\
\hline Barthel $95-100$ at 8 days & $12(57.1 \%)$ & $27(28.7 \%)$ & 3.31 & $1.25-8.78$ & 0.010 \\
\hline Mini mental state ${ }^{\dagger}$ & $25(6)$ & $21(9)$ & & & 0.079 \\
\hline
\end{tabular}

OR: odds ratio; CI: confidence interval; NA: non assessable. ${ }^{*}$ Results are given as mean (SD). ${ }^{\dagger}$ Result is reported as mean (SD) in patients for whom the test was assessable. All other variables did not differ between both groups.

forced exercise have a neuroprotective effect [4], which could result from an improvement of cerebral blood flow through an increase in eNOS expression or angiogenesis or from the modulation of neuroplasticity through nerve growth factor or brain-derived neurotrophic factor expression $[4$, 5]. As it is suggested by the present results, the stroke protection induced by regular physical activity in human might be due to lower heart rate, peak systolic pressure and circulating catecholamine levels [11, 12], improved lipid profile $[11,13]$, and lower levels of arteriosclerosis [14] and inflammation [15], a number of factors that may decrease plaque vulnerability [13]. As in animal models, physical activity in human may have antithrombotic effects through enhancing fibrinolysis and reducing blood viscosity, fibrinogen levels, and platelet aggregation [1].
The improvement of all these biological conditions may also contribute to explain that previous leisure-time physical activity is less likely to be associated with atherosclerosis disease as an etiology of ischemic stroke. Here, we also found that leisure-time physical activity is also less likely to be associated with cardioembolic stroke. Cardioembolic strokes are mainly the consequence of atrial fibrillation of which both incidence and prevalence is increasing with age and the presence of arterial hypertension [16]. The relationship between physical activity and a decrease in the risk to develop an atrial fibrillation has been recently discussed [17], but the presence of other vascular diseases may be confounding factor $[18,19]$. On the other hand, we found that cervical artery dissection is more likely to be the etiology of stroke in patients having prior leisure-time physical activity. Even if 
minor trauma including those produced by physical activity may be implied in the pathophysiology of cervical artery dissection, patients are usually younger and have less or no vascular risk factors [20], a profile that is also more likely to be associated with regular physical activity.

The vascular profile of the subjects may not be the only cornerstone of such a protection that could be observed not only in stroke, but also in various diseases such as cognitive, depressive, or anxiety disorders. Indeed, as it is observed here according to MMS values, regular physical activity may prevent cognitive decline and decrease the risk of vascular dementia [21]. A randomized control trial also demonstrates that a 6-month program of physical activity could afford a modest improvement in cognition over an 18-month followup period in older adults at risk for Alzheimer's disease [22]. In the field of psychiatry, several studies also provide evidence about the ability of physical activity to decrease the incidence of both depression and anxiety disorders as well as to improve depressive and anxious symptoms [23]. In this context, it is of particular interest to underline that the effects of physical activity on the brain can be enhanced by concomitant consumption of natural products such as plant polyphenols or omega-3 fatty acids, some compounds that may share the same mechanisms of action [24,25].

Our study also has limitations. It was a nonrandomized hospital-based study and thus present data should be considered hypothesis generating. Results probably could not be generalized to all patients with ischemic stroke. Because of the observational study design, we cannot rule out that the association between previous leisure-time physical activity and stroke outcome was confounded by other factors that were not taken into account in our analysis such as diet habits.

Because looking for pharmacological agents that may have neuroprotective properties in stroke and other various brain diseases is particularly complex and still remains disappointing, the time has come to optimize the natural resources through education about regular physical activity and also possibly appropriate food intake that may be the easiest and more effective ways to protect the brain.

\section{Funding}

This study was funded by a Programme Hospitalier de Recherche Clinique (PHRC 2001R/1921) and by the research group on "Alzheimer disease and vascular pathologies" (EA 1046) from the Ministère Français de l'Education Nationale, de la Recherche et de la Technologie.

\section{References}

[1] C. D. Lee, A. R. Folsom, and S. N. Blair, "Physical activity and stroke risk: a meta-analysis," Stroke, vol. 34, no. 10, pp. 24752482, 2003.

[2] G. C. Wendel-Vos, A. J. Schuit, E. J. M. Feskens et al., "Physical activity and stroke. A meta-analysis of observational data," International Journal of Epidemiology, vol. 33, no. 4, pp. 787798, 2004.
[3] L. Diep, J. Kwagyan, J. Kurantsin-Mills, R. Weir, and A. JayamTrouth, "Association of physical activity level and stroke outcomes in men and women: a meta-analysis," Journal of Women's Health, vol. 19, no. 10, pp. 1815-1822, 2010.

[4] M. Endres, K. Gertz, U. Lindauer et al., "Mechanisms of stroke protection by physical activity," Annals of Neurology, vol. 54, no. 5, pp. 582-590, 2003.

[5] F. Zhang, Y. Wu, and J. Jia, "Exercise preconditioning and brain ischemic tolerance," Neuroscience, vol. 177, pp. 170-176, 2011.

[6] D. Deplanque, I. Masse, C. Lefebvre, C. Libersa, D. Leys, and R. Bordet, "Prior TIA, lipid-lowering drug use, and physical activity decrease ischemic stroke severity," Neurology, vol. 67, no. 8, pp. 1403-1410, 2006.

[7] L. H. Krarup, T. Truelsen, C. Gluud et al., "Exstroke pilot trial group. Prestroke physical activity is associated with severity and long-term outcome from first-ever stroke," Neurology, vol. 71, pp. 1313-1318, 2008.

[8] N. Stroud, T. M. Mazwi, L. D. Case et al., "Stroke genetics study Investigators. Prestroke physical activity and early functional status after stroke," Journal of Neurology, Neurosurgery \& Psychiatry, vol. 80, pp. 1019-1022, 2009.

[9] R. L. Sacco, R. Gan, B. Boden-Albala et al., "Leisure-time physical activity and ischemic stroke risk. The Northern Manhattan stroke study," Stroke, vol. 29, no. 2, pp. 380-387, 1998.

[10] E. Mostofsky, E. Laier, E. B. Levitant, W. D. Rosamond, G. Schlaug, and M. A. Mittleman, "Physical activity and onset of acute ischemic stroke. The stroke onset study," The American Journal of Epidemiology, vol. 173, pp. 330-336, 2011.

[11] G. Jennings, L. Nelson, P. Nestel et al., "The effects of changes in physical activity on major cardiovascular risk factors, hemodynamics, sympathetic function, and glucose utilization in man: a controlled study of four levels of activity," Circulation, vol. 73, pp. 30-40, 1986.

[12] K. Kohno, H. Matsuoka, K. Takenaka et al., "Depressor effect by exercise training is associated with amelioration of hyperinsulinemia and sympathetic overactivity," Internal Medicine, vol. 39, no. 12, pp. 1013-1019, 2000.

[13] R. Pruchnic, A. Katsiaras, J. He, D. E. Kelley, C. Winters, and B. H. Goodpaster, "Exercise training increases intramyocellular lipid and oxidative capacity in older adults," The American Journal of Physiology, vol. 287, pp. E857-E862, 2004.

[14] M. A. Mittleman and D. S. Siscovick, "Physical exertion as a trigger of myocardial infarction and sudden cardiac death," Cardiology Clinics, vol. 14, no. 2, pp. 263-270, 1996.

[15] E. S. Ford, "Does exercise reduce inflammation? Physical activity and C-reactive protein among U.S. adults," Epidemiology, vol. 13, no. 5, pp. 561-568, 2002.

[16] J. M. Ferro, "Cardioembolic stroke: an update," The Lancet Neurology, vol. 2, no. 3, pp. 177-188, 2003.

[17] B. M. Everett, D. Conen, J. E. Buring, M. V. Moorthy, I. M. Lee, and C. M. Albert, "Physical activity and the risk of incident atrial fibrillation in women," Cardiovascular Quality and Outcomes, vol. 4, pp. 321-327, 2011.

[18] A. Rosengren, P. J. Hauptman, G. Lappas, L. Olsson, L. Wilhelmsen, and K. Swedberg, "Big men and atrial fibrillation: effects of body size and weight gain on risk of atrial fibrillation in men," European Heart Journal, vol. 30, no. 9, pp. 1113-1120, 2009.

[19] D. Conen, U. B. Tedrow, B. A. Koplan, R. J. Glynn, J. E. Buring, and C. M. Albert, "Influence of systolic and diastolic blood pressure on the risk of incident atrial fibrillation in women," Circulation, vol. 119, no. 16, pp. 2146-2152, 2009. 
[20] S. Debette and D. Leys, "Cervical-artery dissections: predisposing factors, diagnosis, and outcome," The Lancet Neurology, vol. 8, no. 7, pp. 668-678, 2009.

[21] G. Ravaglia, P. Forti, A. Lucicesare et al., "Physical activity and dementia risk in the elderly: findings from a prospective Italian study," Neurology, vol. 70, no. 19, pp. 1786-1794, 2008.

[22] N. T. Lautenschlager, K. L. Cox, L. Flicker et al., "Effect of physical activity on cognitive function in older adults at risk for Alzheimer disease: a randomized trial," Journal of the American Medical Association, vol. 300, no. 9, pp. 1027-1037, 2008.

[23] A. Ströhle, "Physical activity, exercise, depression and anxiety disorders," Journal of Neural Transmission, vol. 116, no. 6, pp. 777-784, 2009.

[24] H. van Praag, "Exercise and the brain: something to chew on," Trends in Neurosciences, vol. 32, no. 5, pp. 283-290, 2009.

[25] V. R. Venna, D. Deplanque, C. Allet, K. Belarbi, M. Hamdane, and R. Bordet, "PUFA induce antidepressant-like effects in parallel to structural and molecular changes in the hippocampus," Psychoneuroendocrinology, vol. 34, no. 2, pp. 199-211, 2009. 


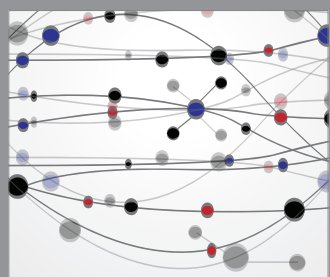

The Scientific World Journal
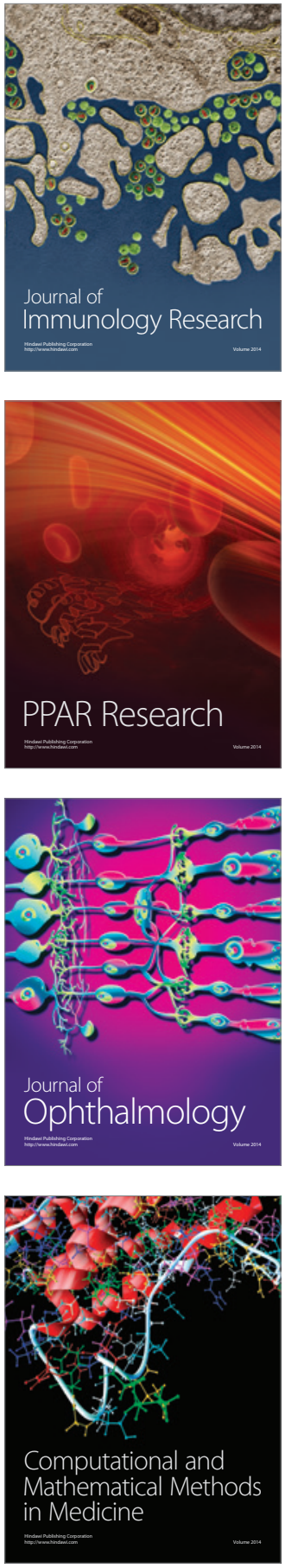

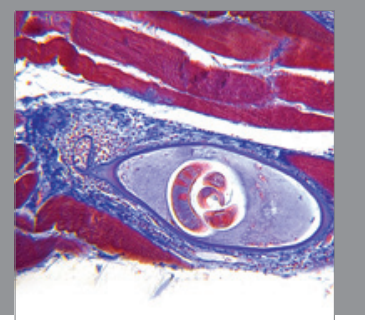

Gastroenterology

Research and Practice
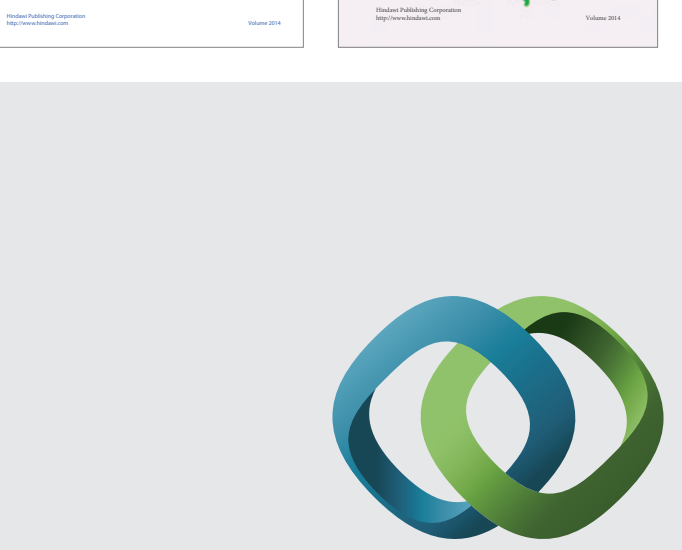

\section{Hindawi}

Submit your manuscripts at

http://www.hindawi.com
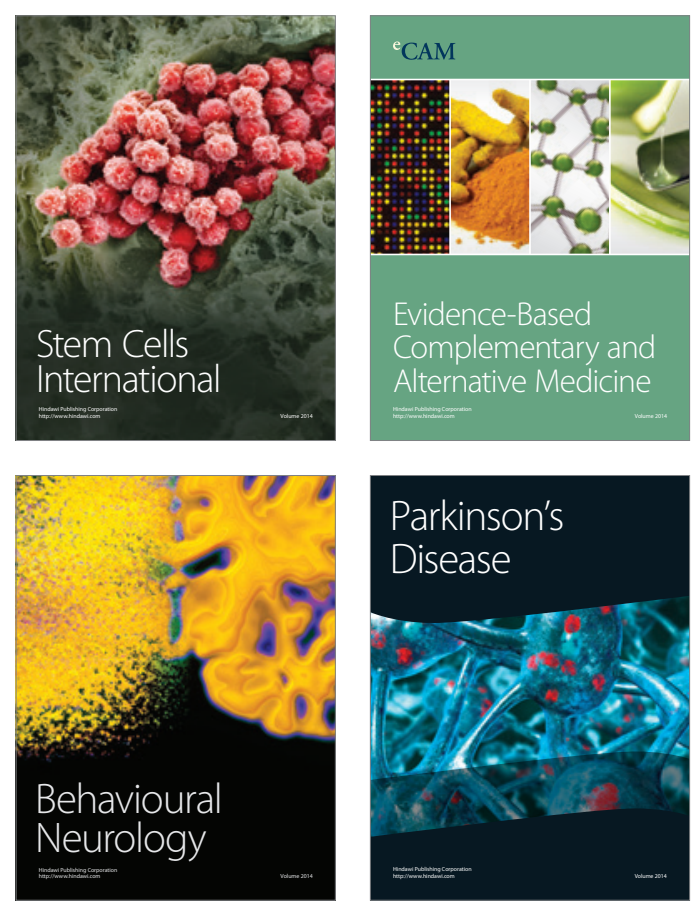

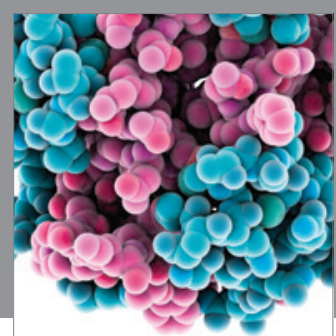

Journal of
Diabetes Research

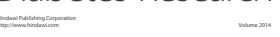

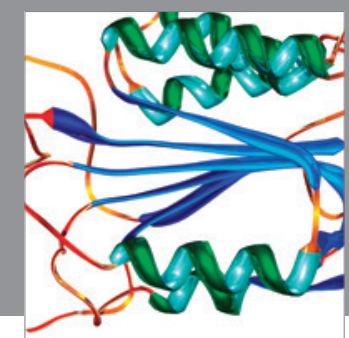

Disease Markers
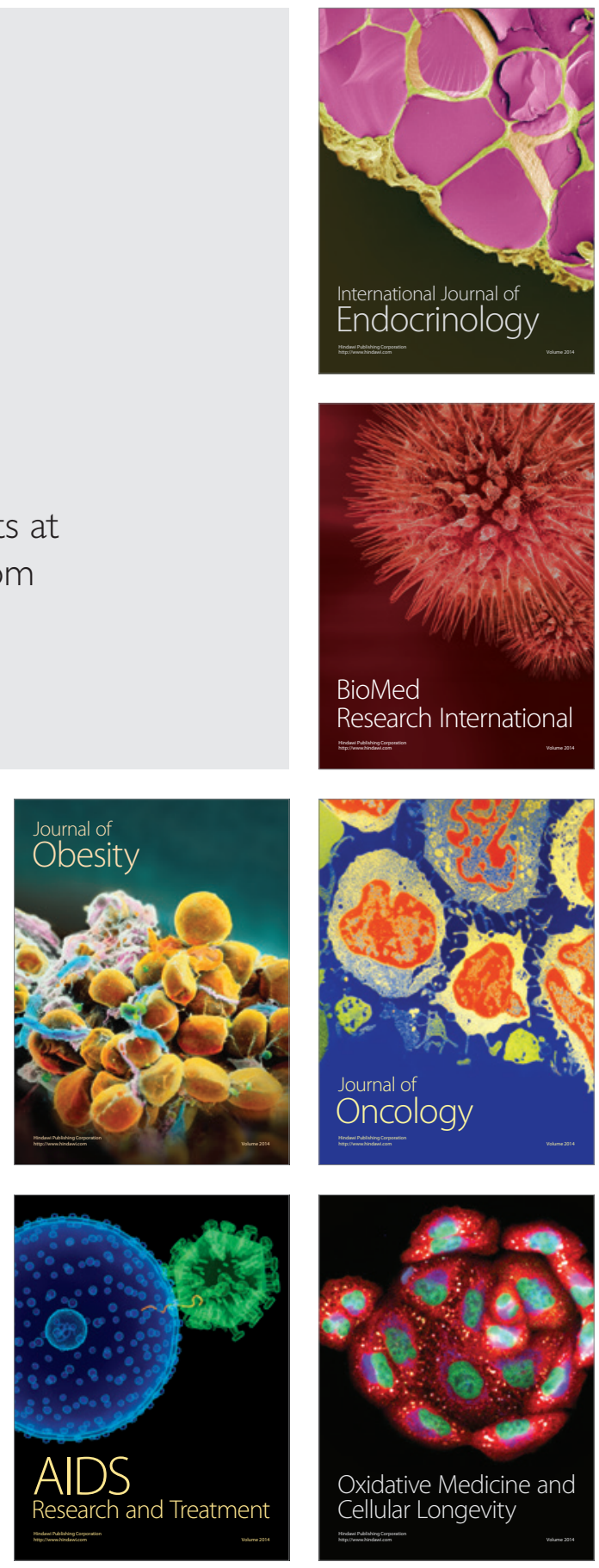